\title{
Extremal functions for Adams' inequalities in dimension four
}

\author{
Xiaomeng $\mathrm{Li}^{1,2}$ \\ ${ }^{1}$ School of Information, Huaibei Normal University, Huaibei, 235000, P. R. China \\ ${ }^{2}$ Department of Mathematics, Renmin University of China, Beijing 100872, P. R. China
}

\begin{abstract}
Let $\Omega \subset \mathbb{R}^{4}$ be a smooth bounded domain, $W_{0}^{2,2}(\Omega)$ be the usual Sobolev space. For any positive integer $\ell, \lambda_{\ell}(\Omega)$ is the $\ell$-th eigenvalue of the bi-Laplacian operator. Define $E_{\ell}=E_{\lambda_{1}(\Omega)} \oplus E_{\lambda_{2}(\Omega)} \oplus$ $\cdots \oplus E_{\lambda_{\ell}(\Omega)}$, where $E_{\lambda_{i}(\Omega)}$ is eigenfunction space associated with $\lambda_{i}(\Omega)$. $E_{\ell}^{\perp}$ denotes the orthogonal complement of $E_{\ell}$ in $W_{0}^{2,2}(\Omega)$. For $0 \leq \alpha<\lambda_{\ell+1}(\Omega)$, we define a norm by $\|u\|_{2, \alpha}^{2}=\|\Delta u\|_{2}^{2}-\alpha\|u\|_{2}^{2}$ for $u \in E_{\ell}^{\perp}$. In this paper, using the blow-up analysis, we prove the following Adams inequalities
\end{abstract}

$$
\sup _{u \in E_{\ell}^{\perp},\|u\|_{2, \alpha} \leq 1} \int_{\Omega} e^{32 \pi^{2} u^{2}} d x<+\infty ;
$$

moreover, the above supremum can be attained by a function $u_{0} \in E_{\ell}^{\perp} \cap C^{4}(\bar{\Omega})$ with $\left\|u_{0}\right\|_{2, \alpha}=1$. This result extends that of Yang (J. Differential Equations, 2015), and complements that of Lu and Yang (Adv. Math. 2009) and Nguyen (arXiv: 1701.08249, 2017).

Keywords: Adams' inequality, Trudinger-Moser inequality, extremal function, blow-up analysis

2000 MSC: 46E35

\section{Introduction}

Trudinger-Moser inequalities play important roles in analysis and geometry. There are two interesting subjects in the study of Trudinger-Moser inequalities: one is the attainability of the best constants, the other is the existence of extremal functions. The research on sharp constants was initiated by Yudovich [42], Pohozaev [31] and Trudinger [34]. Later Moser [29] found the best constant: if $\beta \leq \beta_{0}=n \omega_{n-1}^{1 /(n-1)}$, then

$$
\sup _{u \in W_{0}^{1, n}(\Omega),\|\nabla u\|_{n}=1} \int_{\Omega} e^{\beta|u|^{n /(n-1)}} d x<\infty,
$$

where $\Omega$ is an open subset of $\mathbb{R}^{n}(n \geq 2)$ with finite Lebesgue measure, $\omega_{n-1}$ is the measure of the unit sphere in $\mathbb{R}^{n}$; moreover, if $\beta>\beta_{0}$, the integrals in (1) are still finite, but the supremum is infinite. The sharp constants for higher order derivatives of Moser's inequality was due to

Email address: xmlimath@ruc.edu.cn (Xiaomeng Li ${ }^{1,2}$ )

Preprint submitted to $* * *$

September 23, 2018 
Adams [1]. For any fixed positive integer $m$, let $u \in C_{0}^{m}(\Omega)$, the space of functions having $m$-th continuous derivatives and compact support. To state Adams' result, we use the symbol $\nabla^{m} u$ to denote the $m$-th order gradient for $u$. Precisely

$$
\nabla^{m} u= \begin{cases}\Delta^{\frac{m}{2}} u & \text { when } m \text { is even, } \\ \nabla \Delta^{\frac{m-1}{2}} u & \text { when } m \text { is odd, }\end{cases}
$$

where $\nabla$ and $\Delta$ denote the usual gradient and the Laplacian operators. Adams proved that if $\beta \leq \beta(n, m)$ and $0<m<n$, then

$$
\sup _{u \in W_{0}^{m, \frac{n}{m}}(\Omega),\left\|\nabla^{m} u\right\|_{L^{\frac{n}{m}(\Omega)}} \leq 1} \int_{\Omega} e^{\beta|u|^{n /(n-m)}} d x \leq C_{m, n}|\Omega|
$$

for some constant $C_{m, n}$, where

$$
\beta(n, m)= \begin{cases}\frac{n}{\omega_{n-1}}\left[\frac{\pi^{n / 2} 2^{m} \Gamma\left(\frac{m+1}{2}\right)}{\Gamma\left(\frac{n-m+1}{2}\right)}\right]^{\frac{n}{n-m}} & \text { when } m \text { is odd, } \\ \frac{n}{\omega_{n-1}}\left[\frac{\pi^{n / 2} 2^{m} \Gamma\left(\frac{m}{2}\right)}{\Gamma\left(\frac{n-m}{2}\right)}\right]^{\frac{n}{n-m}} & \text { when } m \text { is even. }\end{cases}
$$

Moreover, $\beta(n, m)$ is the best constant in the sense that if $\beta>\beta(n, m)$, then the supremum in (2) is infinite. The manifold version of Adams' inequality was obtained by Fontana [16].

Extremal functions for (1) were first found by Carleson and Chang [9] when $\Omega$ is the unit ball in $\mathbb{R}^{n}$. This result was then extended by Flucher [15] to a general domain $\Omega \subset \mathbb{R}^{2}$, and by Lin [24] to a bounded smooth domain $\Omega \subset \mathbb{R}^{n}(n \geq 2)$. In 2004, it was proved by Adimurthi and Druet [2] that for any $\alpha, 0 \leq \alpha<\lambda_{1}(\Omega)$, there holds

$$
\sup _{u \in W_{0}^{1,2}(\Omega),\|\nabla u\|_{2} \leq 1} \int_{\Omega} e^{4 \pi^{2}\left(1+\alpha\|u\|_{2}^{2}\right)} d x<+\infty
$$

and the supremum is infinit for $\alpha \geq \lambda_{1}(\Omega)$, where $\lambda_{1}(\Omega)$ is the first eigenvalue of the Laplacian operator with respect to Dirichlet boundary condition. The inequality (3) was generalized by Yang [36] to high dimension, by Lu and Yang [26] and J. Zhu [43] to the versions involving $L^{p}$ norms, by Souza and do Ó [12, 14] and Ruf [32] and Li and Ruf [22] to the whole Euclidean space, by Tintarev [33] and Yang [39] to the following form:

$$
\sup _{u \in W_{0}^{1,2}(\Omega),\|\nabla u\|_{2}^{2}-\alpha\|u\|_{2}^{2} \leq 1} \int_{\Omega} e^{4 \pi u^{2}} d x<+\infty
$$

for any $0 \leq \alpha<\lambda_{1}(\Omega)$. In particular, Yang [39] proved that for $0 \leq \alpha<\lambda_{\ell+1}(\Omega)$, the extremal function for (4) exists, where $\ell$ is a positive integer and $\lambda_{\ell}$ denotes the $\ell$-th eigenvalue of the Laplacian operator with the Dirichlet boundary condition. The singular version of (4) was considered by Yang and Zhu [41], Li and Yang [18], and the author [17] in $\mathbb{R}^{n}(n \geq 2)$. For other works on singular Trudinger-Moser inequalities, we refer the reader to [3, 4, 10, 27] and the references therein.

The study of Turdinger-Moser inequalities on Riemannian manifolds was initiated by Aubin [5] and Cherrier [7, 8]. Much work has also been done in this direction, see for examples [13, 19, 20, 37, 38, 40]. 
Let us come back to the Adams inequality in dimension four. Namely

$$
\sup _{u \in W_{0}^{2,2}(\Omega),\|\Delta u\|_{2} \leq 1} \int_{\Omega} e^{32 \pi^{2} u^{2}} d x<+\infty,
$$

where $\Omega \subset \mathbb{R}^{4}$ is a smooth bounded domain. For any $\alpha$ : $0 \leq \alpha<\lambda_{1}(\Omega)$, it was proved by Lu and Yang [25] that

$$
\sup _{u \in W_{0}^{2,2}(\Omega),\|\Delta u\|_{2}^{2}=1} \int_{\Omega} e^{32 \pi^{2} u^{2}\left(1+\alpha\|u\|_{2}^{2}\right)} d x<+\infty
$$

and the supremum is infinite when $\alpha \geq \lambda_{1}(\Omega)$. Here, by definition,

$$
\lambda_{1}(\Omega)=\inf _{u \in W_{0}^{2,2}(\Omega), u \neq 0} \frac{\|\Delta u\|_{2}^{2}}{\|u\|_{2}^{2}} .
$$

The extremal function for supremum (6) was obtained for $\alpha$ sufficiently small. This result is recently strengthened by Nguyen [30] to the following form:

$$
\sup _{u \in W_{0}^{2,2}(\Omega),\|\Delta u\|_{2}^{2}-\alpha\|u\|_{2}^{2} \leq 1} \int_{\Omega} e^{32 \pi^{2} u^{2}} d x<+\infty
$$

for $0 \leq \alpha<\lambda_{1}(\Omega)$, and the above supremum can be achieved by applying the blow-up analysis method. Motivated by the work [39], we shall improve (7) to the case involving higher order eigenvalues. Note that the Dirichlet boundary problem

$$
\begin{cases}\Delta^{2} u=\lambda u & \text { in } \Omega, \\ u=\frac{\partial u}{\partial v}=0 & \text { on } \partial \Omega\end{cases}
$$

possesses a sequence of eigenvalues $0<\lambda_{1}(\Omega)<\cdots<\lambda_{i}(\Omega)<\lambda_{i+1}(\Omega)<\cdots$. It is known that $\lambda_{i}(\Omega) \rightarrow \infty$ as $i \rightarrow \infty$, see for example ([6], Section 6.3). The corresponding eigenfunction space can be written as

$$
E_{\lambda_{i}(\Omega)}=\left\{u \in W_{0}^{2,2}(\Omega): \Delta^{2} u=\lambda_{i}(\Omega) u\right\} .
$$

For any positive integer $\ell$, we set

$$
E_{\ell}=E_{\lambda_{1}(\Omega)} \oplus E_{\lambda_{2}(\Omega)} \oplus \cdots \oplus E_{\lambda_{\lambda_{\ell}}(\Omega)}
$$

and

$$
E_{\ell}^{\perp}=\left\{u \in W_{0}^{2,2}(\Omega): \int_{\Omega} u v d x=0, \forall v \in E_{\ell}\right\} .
$$

Clearly $W_{0}^{2,2}(\Omega)$ is a real Hilbert space when it is equipped with the inner product

$$
<u, v>=\int_{\Omega} \Delta u \Delta v d x, \quad \forall u, v \in W_{0}^{2,2}(\Omega) .
$$

According to ([6], Theorem 9.31), each eigenfunction space $E_{\lambda_{i}(\Omega)}$ has finite dimension. Suppose $\operatorname{dim} E_{\lambda_{i}(\Omega)}=n_{i}$ and $\left(e_{i j}\right)\left(1 \leq j \leq n_{i}, 1 \leq i \leq \ell\right)$ be the basis of $E_{\ell}$. Then

$$
\left\{\begin{array}{l}
E_{\lambda_{i}(\Omega)}=\operatorname{span}\left\{e_{i 1}, \cdots, e_{i n_{i}}\right\}, \quad i=1,2, \cdots, \ell, \\
E_{\ell}=\operatorname{span}\left\{e_{11}, \cdots, e_{1 n_{1}}, e_{21}, \cdots, e_{2 n_{2}}, \cdots, e_{\ell 1}, \cdots, e_{\ell n_{\ell}}\right\} \\
\int_{\Omega} e_{i j} e_{k l} d x=0 \quad i \neq k \text { or } j \neq l \\
\int_{\Omega} e_{i j}^{2} d x=1
\end{array}\right.
$$


Similar as in [39], we define

$$
\lambda_{\ell+1}(\Omega)=\inf _{u \in W_{0}^{2,2}(\Omega), u \in E_{\ell}^{\perp}, u \neq 0} \frac{\|\Delta u\|_{2}^{2}}{\|u\|_{2}^{2}} .
$$

If $u \in E_{\ell}^{\perp}$ satisfies $\int_{\Omega}|\Delta u|^{2} d x-\alpha \int_{\Omega} u^{2} d x \geq 0$, then we denote

$$
\|u\|_{2, \alpha}=\left(\int_{\Omega}|\Delta u|^{2} d x-\alpha \int_{\Omega} u^{2} d x\right)^{1 / 2} .
$$

In this paper, we prove the following:

Theorem 1. Let $\Omega$ be a smooth bounded domain in $\mathbb{R}^{4}, \ell$ be a positive integer, $E_{\ell}^{\perp}$ and $\lambda_{\ell+1}(\Omega)$ be defined as in (8) and (10) respectively. Then for any $0 \leq \alpha<\lambda_{\ell+1}(\Omega)$, the supremum

$$
\sup _{u \in E_{\ell}^{\perp},\|u\|_{2, \alpha} \leq 1} \int_{\Omega} e^{32 \pi^{2} u^{2}} d x
$$

can be attained by some function $u_{0} \in E_{\ell}^{\perp} \cap C^{4}(\bar{\Omega})$ with $\left\|u_{0}\right\|_{2, \alpha}=1$.

Obviously Theorem—extends a result of Yang ([39], Theorem 2) and includes ([30], Theorem 1.3) as a special case. The proof of Theorem 1 is based on blow-up analysis, which is also used in $[21,25,30]$.

The remaining part of this paper is organized as follows: In section 2, we state some results which are crucial in the subsequent analysis; We prove the existence of subcritical maximizers in section 3 and study the asymptotic behavior of these maximizers in section 4; In section 5, we will give upper bound estimates of the functional $\int_{\Omega} e^{32 \pi^{2} u^{2}} d x$ under the assumption of blow-up analysis; In section 6, we construct a sequence of test functions to complete the proof of Theorem 1

\section{Preliminary results}

In this section, we state some preliminaries which would bring a great convenience during our calculation.

Let $G: \Omega \times \Omega \rightarrow \mathbb{R}$ be the Green function of $\Delta^{2}$ under the Dirichlet condition. That is, for every $y \in \Omega$, the mapping $x \mapsto G(x, y)$ satisfies (in the sense of distribution)

$$
\begin{cases}\Delta^{2} G(x, y)=\delta_{x}(y) & \text { in } \Omega, \\ G(x, y)=\frac{\partial G}{\partial v}=0 & \text { on } \partial \Omega .\end{cases}
$$

All functions $u \in W_{0}^{2,2}(\Omega) \cap C^{4}(\bar{\Omega})$ satisfying $\Delta^{2} u=f(u)$ can be written as

$$
u(x)=\int_{\Omega} G(x, y) f(y) d y .
$$

Now, we collect a property for derivatives of $G$, see for example [11]. There exists $C>0$ such that

$$
|G(x, y)| \leq C \log \left(2+\frac{1}{|x-y|}\right)
$$


and

$$
\left|\nabla^{k} G(x, y)\right| \leq C|x-y|^{-k}, \quad k \geq 1
$$

for all $x, y \in \Omega, x \neq y$. We next recall the Pohozaev identity due to Mitidieri [28].

Lemma 2. Let $\Omega^{\prime}$ be a smooth bounded domain in $\mathbb{R}^{4}, u \in C^{4}\left(\bar{\Omega}^{\prime}\right)$ be a solution of $\Delta^{2} u=f(u)$ in $\Omega^{\prime}$. Then we have for any $y \in \mathbb{R}^{4}$,

$$
\begin{aligned}
4 \int_{\Omega^{\prime}} F(u) d x= & \int_{\partial \Omega^{\prime}}<x-y, v>F(u) d \sigma+\frac{1}{2} \int_{\partial \Omega^{\prime}} v^{2}<x-y, v>d \sigma+2 \int_{\partial \Omega^{\prime}} \frac{\partial u}{\partial v} v d \sigma \\
& +\int_{\partial \Omega^{\prime}}\left(\frac{\partial v}{\partial v}<x-y, D u>+\frac{\partial u}{\partial v}<x-y, D v>-<D v, D u><x-y, v>\right) d \sigma
\end{aligned}
$$

where $F(u)=\int_{0}^{u} f(t) d t,-\Delta u=v$ and $v$ is the normal outward derivative of $x$ on $\partial \Omega^{\prime}$.

Similar to [25, 30], we have the following Lion's type result. Namely

Lemma 3. Let $\left\{u_{k}\right\} \subset E_{\ell}^{\perp}$ be a sequence of functions and $0 \leq \alpha<\lambda_{\ell+1}(\Omega)$ be fixed. If $u_{k} \rightarrow u^{*}$ weakly in $W_{0}^{2,2}(\Omega)$ and $\left\|u_{k}\right\|_{2, \alpha}=1$. Then for any $p<\left(1-\left\|u^{*}\right\|_{2, \alpha}^{2}\right)^{-1}$

$$
\limsup _{k \rightarrow \infty} \int_{\Omega} e^{32 \pi^{2} p u_{k}^{2}} d x<\infty \text {. }
$$

\section{Extremals for subcritical Adams inequalities}

In this section, we shall prove that for any $0<\epsilon<32 \pi^{2}$, there exists some function $u_{\epsilon} \epsilon$ $E_{\ell}^{\perp} \cap C^{4}(\bar{\Omega})$ with $\left\|u_{\epsilon}\right\|_{2, \alpha}=1$ such that

$$
\int_{\Omega} e^{\left(32 \pi^{2}-\epsilon\right) u_{\epsilon}^{2}} d x=\sup _{u \in E_{\ell}^{\perp},\|u\|_{2, \alpha} \leq 1} \int_{\Omega} e^{\left(32 \pi^{2}-\epsilon\right) u^{2}} d x
$$

where $\|\cdot\|_{2, \alpha}$ is defined as in 111 .

This is based on a direct method in calculus of variations. For any $0<\epsilon<32 \pi^{2}$, we take a sequence of functions $\left\{u_{k}\right\} \subset E_{\ell}^{\perp}$ satisfying that

$$
\int_{\Omega}\left|\Delta u_{k}\right|^{2} d x-\alpha \int_{\Omega} u_{k}^{2} d x \leq 1
$$

and that

$$
\lim _{k \rightarrow \infty} \int_{\Omega} e^{\left(32 \pi^{2}-\epsilon\right) u_{k}^{2}} d x=\sup _{u \in E_{\ell}^{+},\|u\|_{2, \alpha} \leq 1} \int_{\Omega} e^{\left(32 \pi^{2}-\epsilon\right) u^{2}} d x .
$$

It follows from (15) and $0 \leq \alpha<\lambda_{\ell+1}(\Omega)$ that $u_{k}$ is bounded in $W_{0}^{2,2}(\Omega)$. Then there exists some function $u_{\epsilon} \in W_{0}^{2,2}(\Omega)$ such that up to a subsequence

$$
\begin{array}{ll}
u_{k} \rightarrow u_{\epsilon} & \text { weakly in } W_{0}^{2,2}(\Omega), \\
u_{k} \rightarrow u_{\epsilon} & \text { strongly in } L^{q}(\Omega)(\forall q>1), \\
u_{k} \rightarrow u_{\epsilon} & \text { a.e. in } \Omega .
\end{array}
$$


Since $u_{k} \in E_{\ell}^{\perp}$, we have

$$
\int_{\Omega} u_{\epsilon} e_{i j} d x=\lim _{k \rightarrow \infty} \int_{\Omega} u_{k} e_{i j} d x=0, \quad \forall 1 \leq j \leq n_{i}, 1 \leq i \leq \ell .
$$

Hence $u_{\epsilon} \in E_{\ell}^{\perp}$. By Lemma 3, we have $e^{\left(32 \pi^{2}-\epsilon\right) u_{k}^{2}}$ is bounded in $L^{r}(\Omega)$ for some $r>1$. Therefore

$$
\lim _{k \rightarrow \infty} \int_{\Omega} e^{\left(32 \pi^{2}-\epsilon\right) u_{k}^{2}} d x=\int_{\Omega} e^{\left(32 \pi^{2}-\epsilon\right) u_{\epsilon}^{2}} d x .
$$

This together with (16) immediately leads to (14). Obviously the supremum (14) is strictly greater than the volume of $\Omega$. Thus $u_{\epsilon} \neq 0$. If $\left\|u_{\epsilon}\right\|_{2, \alpha}<1$, we have

$$
\begin{aligned}
\sup _{u \in E_{\ell}^{\perp},\|u\|_{2, \alpha} \leq 1} \int_{\Omega} e^{\left(32 \pi^{2}-\epsilon\right) u^{2}} d x & =\int_{\Omega} e^{\left(32 \pi^{2}-\epsilon\right) u_{\epsilon}^{2}} d x \\
& <\int_{\Omega} e^{\left(32 \pi^{2}-\epsilon\right) u_{\epsilon}^{2} /\left\|u_{\epsilon}\right\|_{2, \alpha}^{2}} d x \\
& \leq \sup _{u \in E_{\ell}^{\perp},\|u\|_{2, \alpha} \leq 1} \int_{\Omega} e^{\left(32 \pi^{2}-\epsilon\right) u^{2}} d x
\end{aligned}
$$

which is a contradiction. Then we obtain $\left\|u_{\epsilon}\right\|_{2, \alpha}=1$.

A straightforward calculation shows that $u_{\epsilon}$ satisfies the following Euler-Lagrange equation:

$$
\left\{\begin{array}{l}
\Delta^{2} u_{\epsilon}-\alpha u_{\epsilon}=\frac{1}{\lambda_{\epsilon}} u_{\epsilon} e^{\left(32 \pi^{2}-\epsilon\right) u_{\epsilon}^{2}}-\sum_{i=1}^{\ell} \sum_{j=1}^{n_{i}} \frac{\beta_{i j, \epsilon}}{\lambda_{\epsilon}} e_{i j} \quad \text { in } \Omega \\
\int_{\Omega}\left|\Delta u_{\epsilon}\right|^{2} d x-\alpha \int_{\Omega} u_{\epsilon}^{2} d x=1 \\
u_{\epsilon}=\frac{\partial u_{\epsilon}}{\partial v}=0 \text { on } \partial \Omega \\
\lambda_{\epsilon}=\int_{\Omega} u_{\epsilon}^{2} e^{\left(32 \pi^{2}-\epsilon\right) u_{\epsilon}^{2}} d x \\
\beta_{i j, \epsilon}=\int_{\Omega} e_{i j} u_{\epsilon} e^{\left(32 \pi^{2}-\epsilon\right) u_{\epsilon}^{2}} d x
\end{array}\right.
$$

Applying the standard regularity theory to 17 , we obtain $u_{\epsilon} \in C^{4}(\bar{\Omega})$. Since $u_{\epsilon}$ is bounded in $W_{0}^{2,2}(\Omega)$, we can assume without loss of generality,

$$
\begin{array}{ll}
u_{\epsilon} \rightarrow u_{0} & \text { weakly in } W_{0}^{2,2}(\Omega), \\
u_{\epsilon} \rightarrow u_{0} & \text { strongly in } L^{q}(\Omega)(\forall q>1), \\
u_{\epsilon} \rightarrow u_{0} & \text { a.e. in } \Omega .
\end{array}
$$

Since $u_{\epsilon} \in E_{\ell}^{\perp}$, then

$$
\int_{\Omega} u_{0} e_{i j} d x=\lim _{\epsilon \rightarrow 0} \int_{\Omega} u_{\epsilon} e_{i j} d x=0, \quad 1 \leq j \leq n_{i}, 1 \leq i \leq \ell .
$$

Hence we get $u_{0} \in E_{\ell}^{\perp}$.

Denote $c_{\epsilon}=\left|u_{\epsilon}\right|\left(x_{\epsilon}\right)=\max _{\Omega}\left|u_{\epsilon}\right|$. If $c_{\epsilon}$ is bounded, then $e^{\left(32 \pi^{2}-\epsilon\right) u_{\epsilon}^{2}}$ is bounded in $L^{\infty}(\Omega)$. Clearly $\lambda_{\epsilon}^{-1} \beta_{i j, \epsilon} e_{i j}$ is also bounded in $L^{\infty}(\Omega)$. Thus for any $u \in E_{\ell}^{\perp}(\Omega)$ with $\|u\|_{2, \alpha} \leq 1$, we have by (14) and the Lebesgue dominated convergence theorem

$$
\int_{\Omega} e^{32 \pi^{2} u^{2}} d x=\lim _{\epsilon \rightarrow 0} \int_{\Omega} e^{\left(32 \pi^{2}-\epsilon\right) u^{2}} d x \leq \lim _{\epsilon \rightarrow 0} \int_{\Omega} e^{\left(32 \pi^{2}-\epsilon\right) u_{\epsilon}^{2}} d x=\int_{\Omega} e^{32 \pi^{2} u_{0}^{2}} d x .
$$


This implies that

$$
\int_{\Omega} e^{32 \pi^{2} u_{0}^{2}} d x=\sup _{u \in E_{\ell}^{\perp},\|u\|_{2, \alpha} \leq 1} \int_{\Omega} e^{32 \pi^{2} u^{2}} d x .
$$

Obviously $\left\|u_{0}\right\|_{2, \alpha}=1$. Using the inequality $e^{t} \leq 1+t e^{t}$ for $t \geq 0$, we have

$$
\begin{aligned}
\int_{\Omega} e^{\left(32 \pi^{2}-\epsilon\right) u_{\epsilon}^{2}} d x & \leq \int_{\Omega}\left(1+\left(32 \pi^{2}-\epsilon\right) u_{\epsilon}^{2} e^{\left(32 \pi^{2}-\epsilon\right) u_{\epsilon}^{2}}\right) d x \\
& =|\Omega|+\left(32 \pi^{2}-\epsilon\right) \lambda_{\epsilon}
\end{aligned}
$$

Hence

$$
\liminf _{\epsilon \rightarrow 0} \lambda_{\epsilon}>0
$$

Applying the standard regularity theory to (17), we obtain $u_{0} \in C^{4}(\bar{\Omega})$. Therefore $u_{0}$ is a desired extremal function.

Without loss of generality, we assume there exists some point $x_{0} \in \bar{\Omega}$ such that

$$
x_{\epsilon} \rightarrow x_{0}, \quad c_{\epsilon}=u_{\epsilon}\left(x_{\epsilon}\right) \rightarrow+\infty \quad \text { as } \quad \epsilon \rightarrow 0
$$

or we will replace $u_{\epsilon}$ by $-u_{\epsilon}$ instead. In the sequel, we do not distinguish sequence and subsequence, the reader can understand it from the context.

\section{Asymptotic behavior of extremals for subcritical functionals}

In this section, we shall prove that $u_{0}=0$ and obtain the following Lions type energy concentration result:

$$
\left|\Delta u_{\epsilon}\right|^{2} d x \rightarrow \delta_{x_{0}} \quad \text { as } \quad \epsilon \rightarrow 0
$$

in the sense of measure, where $\delta_{x_{0}}$ is the usual Dirac measure centered at $x_{0}$.

Suppose $u_{0} \not \equiv 0$. In view of Lemma 3 , we have $e^{\left(32 \pi^{2}-\epsilon\right) u_{\epsilon}^{2}}$ is bounded in $L^{r}(\Omega)$ for any fixed $r$ with $1<r<1 /\left(1-\left\|u_{0}\right\|_{2, \alpha}^{2}\right)$. Note also that $\lambda_{\epsilon}^{-1} \beta_{i j, \epsilon} e_{i j}$ is bounded in $L^{\infty}(\Omega)$. Applying the standard regularity theory to 17 , we get $u_{\epsilon}$ is uniformly bounded in $\Omega$, which contradicts with $c_{\epsilon} \rightarrow \infty$ as $\epsilon \rightarrow 0$. Hence $u_{0} \equiv 0$.

Similar to [25, 30], we can derive $x_{0} \notin \partial \Omega$. When $x_{0} \in \Omega$, suppose (18) is not true. Noting that $\left\|\Delta u_{\epsilon}\right\|_{2, \alpha}=1$, we can find $r_{0}>0$ and $0<\eta<1$ such that

$$
\limsup _{\epsilon \rightarrow 0} \int_{\mathbb{B}_{r_{0}}\left(x_{0}\right)}\left|\Delta u_{\epsilon}\right|^{2} d x \leq 1-\eta .
$$

Choose a cut-off function $\phi \in C_{0}^{2}\left(\mathbb{B}_{r_{0}}\left(x_{0}\right)\right)$, which is equal to 1 on $\mathbb{B}_{r_{0} / 2}\left(x_{0}\right)$, such that $\mathbb{B}_{r_{0}}\left(x_{0}\right) \subset \Omega$ and

$$
\limsup _{\epsilon \rightarrow 0} \int_{\mathbb{B}_{r_{0}}\left(x_{0}\right)}\left|\Delta\left(\phi u_{\epsilon}\right)\right|^{2} d x \leq 1-\eta .
$$

By the Adams inequality (5), $e^{\left(32 \pi^{2}-\epsilon\right) \phi^{2} u_{\epsilon}^{2}}$ is bounded in $L^{r}\left(\mathbb{B}_{r_{0}}\left(x_{0}\right)\right)$ for some $r>1$ and thus $e^{\left(32 \pi^{2}-\epsilon\right) u_{\epsilon}^{2}}$ is bounded in $L^{r}\left(\mathbb{B}_{r_{0} / 2}\left(x_{0}\right)\right)$ provided that $\epsilon$ is sufficiently small. On the other hand, $\lambda_{\epsilon}^{-1} \beta_{i j, \epsilon} e_{i j}$ is bounded in $L^{\infty}(\Omega)$. Applying the standard regularity theory to [17), we derive that $u_{\epsilon}$ is bounded in $\overline{\mathbb{B}_{r_{0} / 4}\left(x_{0}\right)}$ contradicting $c_{\epsilon} \rightarrow \infty$. Hence we obtain (18). 
Let

$$
r_{\epsilon}^{4}=\frac{\lambda_{\epsilon}}{c_{\epsilon}^{2} e^{\left(32 \pi^{2}-\epsilon\right) c_{\epsilon}^{2}}} .
$$

Then for any $0<\gamma<32 \pi^{2}$, we have by the Hölder inequality and the Adams inequality (5),

$$
\lim _{\epsilon \rightarrow 0} r_{\epsilon}^{4} c_{\epsilon}^{2} e^{\gamma c_{\epsilon}^{2}}=\lim _{\epsilon \rightarrow 0} e^{-\left(32 \pi^{2}-\epsilon-\gamma\right) c_{\epsilon}^{2}} \int_{\Omega} u_{\epsilon}^{2} e^{\left(32 \pi^{2}-\epsilon\right) u_{\epsilon}^{2}} d x=0 .
$$

This implies that $r_{\epsilon}$ converges to zero rapidly. To proceed, we set the following quantities

$$
b_{\epsilon}=\frac{\lambda_{\epsilon}}{\int_{\Omega}\left|u_{\epsilon}\right| e^{\left(32 \pi^{2}-\epsilon\right) u_{\epsilon}^{2}} d x}, \quad \vartheta=\lim _{\epsilon \rightarrow 0} \frac{c_{\epsilon}}{b_{\epsilon}}, \quad \mu=\lim _{\epsilon \rightarrow 0} \frac{\int_{\Omega} u_{\epsilon} e^{\left(32 \pi^{2}-\epsilon\right) u_{\epsilon}^{2}} d x}{\int_{\Omega}\left|u_{\epsilon}\right| e^{\left(32 \pi^{2}-\epsilon\right) u_{\epsilon}^{2}} d x} .
$$

Let $\Omega_{\epsilon}=\left\{x \in \mathbb{R}^{4}: x_{\epsilon}+r_{\epsilon} x \in \Omega\right\}$. Define two blow-up sequences of functions on $\Omega_{\epsilon}$ as

$$
v_{\epsilon}(x)=\frac{u_{\epsilon}\left(x_{\epsilon}+r_{\epsilon} x\right)}{c_{\epsilon}}, \quad w_{\epsilon}(x)=b_{\epsilon}\left(u_{\epsilon}\left(x_{\epsilon}+r_{\epsilon} x\right)-c_{\epsilon}\right) .
$$

A straightforward calculation shows

$$
\Delta^{2} v_{\epsilon}(x)=\alpha r_{\epsilon}^{4} v_{\epsilon}(x)+\lambda_{\epsilon}^{-1} r_{\epsilon}^{4} v_{\epsilon}(x) e^{\left(32 \pi^{2}-\epsilon\right) u_{\epsilon}^{2}\left(x_{\epsilon}+r_{\epsilon} x\right)}-c_{\epsilon}^{-1} r_{\epsilon}^{4} \sum_{i=1}^{\ell} \sum_{j=1}^{n_{i}} \frac{\beta_{i j, \epsilon}}{\lambda_{\epsilon}} e_{i j} \quad \text { in } \quad \Omega_{\epsilon}
$$

and

$$
\Delta^{2} w_{\epsilon}(x)=\alpha b_{\epsilon} c_{\epsilon} r_{\epsilon}^{4} v_{\epsilon}(x)+b_{\epsilon} c_{\epsilon}^{-1} v_{\epsilon}(x) e^{\left(32 \pi^{2}-\epsilon\right) c_{\epsilon} b_{\epsilon}^{-1}\left(1+v_{\epsilon}\right) w_{\epsilon}}-b_{\epsilon} r_{\epsilon}^{4} \sum_{i=1}^{\ell} \sum_{j=1}^{n_{i}} \frac{\beta_{i j, \epsilon}}{\lambda_{\epsilon}} e_{i j} \quad \text { in } \quad \Omega_{\epsilon} .
$$

Obviously $\left|v_{\epsilon}\right| \leq 1$. Then for any fixed $R>0$ and $x \in \mathbb{B}_{R}(0)$, we obtain

$$
\int_{\mathbb{B}_{R}(0)}\left|\Delta v_{\epsilon}\right|^{2} d x=\int_{\mathbb{B}_{R r_{\epsilon}}\left(x_{\epsilon}\right)} \frac{\left|\Delta u_{\epsilon}(y)\right|^{2}}{c_{\epsilon}^{2}} d y=o_{\epsilon}(1)
$$

and

$$
\left|\Delta^{2} v_{\epsilon}(x)\right|=\left|r_{\epsilon}^{4}\left(\alpha v_{\epsilon}(x)+\lambda_{\epsilon}^{-1} v_{\epsilon} e^{\left(32 \pi^{2}-\epsilon\right) u_{\epsilon}^{2}\left(x_{\epsilon}+r_{\epsilon} x\right)}-c_{\epsilon}^{-1} \sum_{i=1}^{\ell} \sum_{j=1}^{n_{i}} \frac{\beta_{i j, \epsilon}}{\lambda_{\epsilon}} e_{i j}\right)\right|=o_{\epsilon}(1) .
$$

These estimates and the standard regularity theory imply

$$
v_{\epsilon} \rightarrow v \quad \text { in } C_{\mathrm{loc}}^{4}\left(\mathbb{R}^{4}\right) .
$$

Since $v(0)=\lim _{\epsilon \rightarrow 0} v_{\epsilon}(0)=1$, we conclude that $v(x) \equiv 1$ on $\mathbb{R}^{4}$ by using the Liouville theorem.

Now we consider the convergence of $w_{\epsilon}$. Using the Green representation formula (12), we get

$$
u_{\epsilon}(x)=\int_{\Omega} G(x, y)\left(\alpha u_{\epsilon}(y)+\frac{1}{\lambda_{\epsilon}} u_{\epsilon}(y) e^{\left(32 \pi^{2}-\epsilon\right) u_{\epsilon}^{2}(y)}-\sum_{i=1}^{\ell} \sum_{j=1}^{n_{i}} \frac{\beta_{i j, \epsilon}}{\lambda_{\epsilon}} e_{i j}\right) d y .
$$


Then for $m=1,2$

$$
\nabla^{m} u_{\epsilon}(x)=\int_{\Omega} \nabla_{x}^{m} G(x, y)\left(\alpha u_{\epsilon}(y)+\frac{1}{\lambda_{\epsilon}} u_{\epsilon}(y) e^{\left(32 \pi^{2}-\epsilon\right) u_{\epsilon}^{2}(y)}-\sum_{i=1}^{\ell} \sum_{j=1}^{n_{i}} \frac{\beta_{i j, \epsilon}}{\lambda_{\epsilon}} e_{i j}\right) d y .
$$

By 13 and (19), we have for any $R>0$ and $x \in \mathbb{B}_{R}(0)$

$$
\begin{aligned}
\left|\nabla^{m} w_{\epsilon}(x)\right|= & b_{\epsilon} r_{\epsilon}^{m}\left|\int_{\Omega} \nabla_{x}^{m} G\left(x_{\epsilon}+r_{\epsilon} x, y\right) \Delta^{2} u_{\epsilon}(y) d y\right| \\
\leq & C b_{\epsilon} r_{\epsilon}^{m}\left(\alpha \int_{\Omega} \frac{|u(y)|}{\left|x_{\epsilon}+r_{\epsilon} x-y\right|^{m}} d y+\frac{1}{\lambda_{\epsilon}} \int_{\mathbb{B}_{2 R_{\epsilon}\left(x_{\epsilon}\right)}} \frac{\left|u_{\epsilon}(y)\right| e^{\left(32 \pi^{2}-\epsilon\right) u_{\epsilon}^{2}(y)}}{\left|x_{\epsilon}+r_{\epsilon} x-y\right|^{m}} d y\right. \\
& \left.+\frac{1}{\lambda_{\epsilon}} \int_{\Omega \mid \mathbb{B}_{2 R r_{\epsilon}\left(x_{\epsilon}\right)}} \frac{\left|u_{\epsilon}(y)\right| e^{\left(32 \pi^{2}-\epsilon\right) u_{\epsilon}^{2}(y)}}{\left|x_{\epsilon}+r_{\epsilon} x-y\right|^{m}} d y+\frac{1}{\lambda_{\epsilon}} \int_{\Omega} \sum_{i=1}^{\ell} \sum_{j=1}^{n_{i}} \frac{\left|\beta_{i j, \epsilon}\right|\left|e_{i j}\right|}{\left|x_{\epsilon}+r_{\epsilon} x-y\right|^{m}} d y\right) \\
\leq & C\left(\alpha b_{\epsilon} c_{\epsilon} r_{\epsilon}^{m} \int_{\Omega} \frac{1}{\left|x_{\epsilon}+r_{\epsilon} x-y\right|^{m}} d y+\frac{b_{\epsilon}}{c_{\epsilon}} \int_{\mathbb{B}_{2 R}(0)} \frac{1}{|x-z|^{m}} d z+\frac{1}{R^{m}}\right. \\
& \left.+\mu r_{\epsilon}^{m} \sum_{i=1}^{\ell} \sum_{j=1}^{n_{i}} \int_{\Omega} \frac{1}{\left|x_{\epsilon}+r_{\epsilon} x-y\right|^{m}} d y\right) \\
\leq & C(R) .
\end{aligned}
$$

Here we have used $b_{\epsilon} \leq c_{\epsilon}$ and $|\mu| \leq 1$. Applying the standard regularity theory to 20, we obtain

$$
w_{\epsilon} \rightarrow w \text { in } C_{\mathrm{loc}}^{4}\left(\mathbb{R}^{4}\right) \text {. }
$$

If $\vartheta=\lim _{\epsilon \rightarrow 0} \frac{c_{\epsilon}}{b_{\epsilon}}<+\infty$, then we can see from (19), 210 and 23) that $w$ satisfies

$$
\left\{\begin{array}{l}
\Delta^{2} w(x)=\frac{1}{\vartheta} e^{64 \pi^{2} \vartheta w(x)} \quad \text { in } \quad \mathbb{R}^{4}, \\
w(x) \leq w(0)=0 \\
\int_{\mathbb{R}^{4}} e^{64 \pi^{2} \vartheta w(x)} d x<+\infty .
\end{array}\right.
$$

To understand $w_{\epsilon}(x)$ further, we have

$$
\Delta w_{\epsilon}=b_{\epsilon} r_{\epsilon}^{2} \int_{\Omega} \Delta_{x} G\left(x_{\epsilon}+r_{\epsilon} x, y\right)\left(\alpha u_{\epsilon}(y)+\frac{1}{\lambda_{\epsilon}} u_{\epsilon}(y) e^{\left(32 \pi^{2}-\epsilon\right) u_{\epsilon}^{2}(y)}-\sum_{i=1}^{\ell} \sum_{j=1}^{n_{i}} \frac{\beta_{i j, \epsilon}}{\lambda_{\epsilon}} e_{i j}\right) d y .
$$

Hence for any $R>0$, we obtain by Fubini theorem

$$
\begin{aligned}
\int_{\mathbb{B}_{R}(0)}\left|\Delta w_{\epsilon}(x)\right| d x \leq & C b_{\epsilon} r_{\epsilon}^{2} \int_{\Omega} \frac{\left|u_{\epsilon}(y)\right| e^{\left(32 \pi^{2}-\epsilon\right) u_{\epsilon}^{2}(y)}}{\lambda_{\epsilon}}\left(\int_{\mathbb{B}_{R}(0)} \frac{1}{\left|x_{\epsilon}+r_{\epsilon} x-y\right|^{2}} d x\right) d y \\
& +C b_{\epsilon} r_{\epsilon}^{2} \int_{\Omega}\left|u_{\epsilon}(y)\right|\left(\int_{\mathbb{B}_{R}(0)} \frac{1}{\left|x_{\epsilon}+r_{\epsilon} x-y\right|^{2}} d x\right) d y \\
& +C b_{\epsilon} r_{\epsilon}^{2} \sum_{i=1}^{\ell} \sum_{j=1}^{n_{i}} \int_{\Omega}\left|e_{i j}\right|\left(\int_{\mathbb{B}_{R}(0)} \frac{1}{\left|x_{\epsilon}+r_{\epsilon} x-y\right|^{2}} d x\right) d y \\
\leq & C R^{2} .
\end{aligned}
$$


This together with 24) and the result of [23, 35] implies that

$$
w(x)=-\frac{1}{16 \pi^{2} \vartheta} \log \left(1+\frac{\pi}{\sqrt{6}}|x|^{2}\right), \quad x \in \mathbb{R}^{4} .
$$

When $\vartheta=+\infty$, we have by $(\underline{22}),|\Delta w(x)| \leq C R^{-2}$ for $x \in \mathbb{B}_{R}(0)$. Letting $R \rightarrow+\infty$, we have $w(x)$ is a harmonic function in $\mathbb{R}^{4}$. Since $w(x) \leq w(0)=0$, then $w(x) \equiv 0$ by the Liouville Theorem.

We next consider the convergence behavior of $u_{\epsilon}$ away from the blow-up point $x_{0}$. Let $\psi_{\epsilon}$ be a solution of the following Dirichlet problem

$$
\left\{\begin{array}{l}
\Delta^{2} \psi_{\epsilon}(x)=\frac{1}{\lambda_{\epsilon}} b_{\epsilon} u_{\epsilon}(x) e^{\left(32 \pi^{2}-\epsilon\right) u_{\epsilon}^{2}(x)}-\sum_{i=1}^{\ell} \sum_{j=1}^{n_{i}} \frac{1}{\lambda_{\epsilon}} b_{\epsilon} \beta_{i j, \epsilon} e_{i j} \quad \text { in } \quad \Omega, \\
\psi_{\epsilon}(x)=\frac{\partial \psi_{\epsilon}}{\partial v}=0 \quad \text { on } \quad \partial \Omega .
\end{array}\right.
$$

Using the Green representation formula (12), we have

$$
\psi_{\epsilon}(x)=\int_{\Omega} G(x, y)\left(\frac{b_{\epsilon}}{\lambda_{\epsilon}} u_{\epsilon}(y) e^{\left(32 \pi^{2}-\epsilon\right) u_{\epsilon}^{2}(y)}-\sum_{i=1}^{\ell} \sum_{j=1}^{n_{i}} \frac{b_{\epsilon} \beta_{i j, \epsilon}}{\lambda_{\epsilon}} e_{i j}\right) d y .
$$

By differentiating with respect to $x$ for $m=1,2$, we obtain

$$
\left|\nabla^{m} \psi_{\epsilon}(x)\right| \leq C\left(\int_{\Omega}|x-y|^{-m} \frac{b_{\epsilon}}{\lambda_{\epsilon}}\left|u_{\epsilon}(y)\right| e^{\left(32 \pi^{2}-\epsilon\right) u_{\epsilon}^{2}(y)} d y+\sum_{i=1}^{\ell} \sum_{j=1}^{n_{i}} \int_{\Omega}|x-y|^{-m} \frac{b_{\epsilon}}{\lambda_{\epsilon}}\left|e_{i j}\right|\left|\beta_{i j, \epsilon}\right| d y\right) .
$$

For $1<s<2$, applying the basic inequality $(a+b)^{s} \leq 2^{s-1}\left(a^{s}+b^{s}\right)$ for $a \geq 0$ and $b \geq 0$ and the Hölder inequality, we obtain

$$
\begin{aligned}
\left|\nabla^{m} \psi_{\epsilon}(x)\right|^{s} & \leq C^{s} 2^{s-1}\left(\int_{\Omega} \frac{b_{\epsilon}}{\lambda_{\epsilon}}\left|u_{\epsilon}(y)\right| \frac{e^{\left(32 \pi^{2}-\epsilon\right) u_{\epsilon}^{2}(y)}}{|x-y|^{m}} d y\right)^{r}+C^{s} 2^{s-1}\left(\sum_{i=1}^{\ell} \sum_{j=1}^{n_{i}} \int_{\Omega} \frac{b_{\epsilon}}{\lambda_{\epsilon}} \frac{\left|e_{i j}\right|\left|\beta_{i j, \epsilon}\right|}{|x-y|^{m}} d y\right)^{r} \\
& \leq C \int_{\Omega} \frac{b_{\epsilon}}{\lambda_{\epsilon}} \frac{\left|u_{\epsilon}(y)\right| e^{\left(32 \pi^{2}-\epsilon\right) u_{\epsilon}^{2}(y)}}{|x-y|^{m s}} d y+C \sum_{i=1}^{\ell} \sum_{j=1}^{n_{i}} \int_{\Omega} \frac{\left|e_{i j}\right|}{|x-y|^{m s}} d y .
\end{aligned}
$$

By the Fubini theorem, we have $\left\|\nabla^{m} \psi_{\epsilon}\right\|_{s} \leq C$ for $m=1,2$. Hence

$$
\left\|\psi_{\epsilon}\right\|_{W_{0}^{2, s}(\Omega)} \leq C .
$$

Denote $\varphi_{\epsilon}=b_{\epsilon} u_{\epsilon}-\psi_{\epsilon}$. In view of (17) and (25), we get

$$
\begin{cases}\Delta^{2} \varphi_{\epsilon}(x)=\alpha\left(\varphi_{\epsilon}(x)+\psi_{\epsilon}(x)\right) & \text { in } \Omega \\ \varphi(x)=\frac{\partial \varphi_{\epsilon}}{\partial v}=0 & \text { on } \partial \Omega\end{cases}
$$

Multiplying both sides of (27) by $\varphi_{\epsilon}$, we have by the definition of $\lambda_{\ell+1}(\Omega)$ and the Hölder inequality,

$$
\begin{aligned}
\int_{\Omega}\left|\Delta \varphi_{\epsilon}\right|^{2} d x & =\alpha \int_{\Omega} \varphi_{\epsilon}^{2} d x+\alpha \int_{\Omega} \psi_{\epsilon} \varphi_{\epsilon} d x \\
& \leq \frac{\alpha}{\lambda_{\ell+1}(\Omega)} \int_{\Omega}\left|\Delta \varphi_{\epsilon}\right|^{2} d x+\frac{\alpha}{\sqrt{\lambda_{\ell+1}(\Omega)}}\left(\int_{\Omega} \psi_{\epsilon}^{2} d x\right)^{1 / 2}\left(\int_{\Omega}\left|\Delta \varphi_{\epsilon}\right|^{2} d x\right)^{1 / 2} .
\end{aligned}
$$


Then we get

$$
\int_{\Omega}\left|\Delta \varphi_{\epsilon}\right|^{2} d x \leq \frac{\alpha^{2} \lambda_{\ell+1}(\Omega)}{\left(\lambda_{\ell+1}(\Omega)-\alpha\right)^{2}} \int_{\Omega} \psi_{\epsilon}^{2} d x .
$$

Hence $\left\|\varphi_{\epsilon}\right\|_{W_{0}^{2,2}(\Omega)} \leq C$. This together with (26) implies that $b_{\epsilon} u_{\epsilon}$ is bounded in $W_{0}^{2, s}(\Omega)$. So we can assume there exists some function $G \in W_{0}^{2, s}(\Omega)$ such that $b_{\epsilon} u_{\epsilon} \rightarrow G$ weakly in $W_{0}^{2, s}(\Omega)$ for any $1<s<2$. Multiplying both sides of (17) by $b_{\epsilon}$, we have

$$
\left\{\begin{array}{l}
\Delta^{2}\left(b_{\epsilon} u_{\epsilon}\right)=\alpha\left(b_{\epsilon} u_{\epsilon}\right)+\frac{1}{\lambda_{\epsilon}}\left(b_{\epsilon} u_{\epsilon}\right) e^{\left(32 \pi^{2}-\epsilon\right) u_{\epsilon}^{2}}-\sum_{i=1}^{\ell} \sum_{j=1}^{n_{i}} \frac{\beta_{i j, \epsilon}}{\lambda_{\epsilon}} b_{\epsilon} e_{i j} \quad \text { in } \Omega, \\
b_{\epsilon} u_{\epsilon}=\frac{\partial\left(b_{\epsilon} u_{\epsilon}\right)}{\partial v}=0 \quad \text { on } \partial \Omega .
\end{array}\right.
$$

For any fixed $r>0$ such that $\mathbb{B}_{r}\left(x_{0}\right) \subset \Omega$, we derive from the Adams inequality (5) and the cut-off function theory that $e^{\left(32 \pi^{2}-\epsilon\right) u_{\epsilon}^{2}}$ is bounded in $L^{p}\left(\Omega \backslash \mathbb{B}_{r}\left(x_{0}\right)\right)$ for some $p>1$. On the other hand, $\lambda^{-1} \beta_{i j, \epsilon} b_{\epsilon} e_{i j}$ is bounded in $L^{\infty}(\Omega)$. Applying the standard regularity theory we infer that

$$
b_{\epsilon} u_{\epsilon} \rightarrow G \quad \text { in } \quad C_{\mathrm{loc}}^{4}\left(\bar{\Omega} \backslash\left\{x_{0}\right\}\right)
$$

For any $\phi \in C^{\infty}(\bar{\Omega})$, we have

$$
\begin{aligned}
& \int_{\Omega} \phi\left(\frac{b_{\epsilon} u_{\epsilon}}{\lambda_{\epsilon}} e^{\left(32 \pi^{2}-\epsilon\right) u_{\epsilon}^{2}}+\alpha b_{\epsilon} u_{\epsilon}-\sum_{i=1}^{\ell} \sum_{j=1}^{n_{i}} \frac{\beta_{i j, \epsilon}}{\lambda_{\epsilon}} b_{\epsilon} e_{i j}\right) d x \\
& =\int_{\Omega}\left(\phi(x)-\phi\left(x_{0}\right)\right) \frac{b_{\epsilon} u_{\epsilon}}{\lambda_{\epsilon}} e^{\left(32 \pi^{2}-\epsilon\right) u_{\epsilon}^{2}} d x+\int_{\Omega} \phi\left(x_{0}\right) \frac{b_{\epsilon} u_{\epsilon}}{\lambda_{\epsilon}} e^{\left(32 \pi^{2}-\epsilon\right) u_{\epsilon}^{2}} d x \\
& \quad+\alpha \int_{\Omega} \phi b_{\epsilon} u_{\epsilon} d x-\sum_{i=1}^{\ell} \sum_{j=1}^{n_{i}} \int_{\Omega} \frac{\beta_{i j, \epsilon}}{\lambda_{\epsilon}} \phi b_{\epsilon} e_{i j} d x
\end{aligned}
$$

Lebesgue dominated convergence theorem implies that

$$
\lim _{\epsilon \rightarrow 0} \int_{\left\{x \in \Omega:,\left|u_{\epsilon}\right| \leq 1\right\}} e^{\left(32 \pi^{2}-\epsilon\right) u_{\epsilon}^{2}} d x=|\Omega| .
$$

Combining (16) and 29], we get

$$
\begin{aligned}
\liminf _{\epsilon \rightarrow 0} \int_{\Omega}\left|u_{\epsilon}\right| e^{\left(32 \pi^{2}-\epsilon\right) u_{\epsilon}^{2}} d x & \geq \liminf _{\epsilon \rightarrow 0} \int_{\left\{x \in \Omega:\left|u_{\epsilon}\right|>1\right\}} e^{\left(32 \pi^{2}-\epsilon\right) u_{\epsilon}^{2}} d x \\
& =\sup _{u \in E_{\ell}^{+},\|u\|_{2, \alpha} \leq 1} \int_{\Omega} e^{32 \pi^{2} u^{2}} d x-|\Omega| \\
& >0 .
\end{aligned}
$$

This leads to the fact that $b_{\epsilon} / \lambda_{\epsilon}$ is bounded. Using the Hölder inequality, we have

$$
\begin{aligned}
& \int_{\Omega \backslash \mathbb{B}_{r}\left(x_{0}\right)}\left(\phi(x)-\phi\left(x_{0}\right)\right) \frac{b_{\epsilon} u_{\epsilon}}{\lambda_{\epsilon}} e^{\left(32 \pi^{2}-\epsilon\right) u_{\epsilon}^{2}} d x \\
\leq & C \max _{x \in \bar{\Omega}} \phi(x)\left(\int_{\Omega \backslash \mathbb{B}_{r}\left(x_{0}\right)} e^{\left(32 \pi^{2}-\epsilon\right) p_{1} u_{\epsilon}^{2}} d x\right)^{1 / p_{1}}\left(\int_{\Omega \backslash \mathbb{B}_{r}\left(x_{0}\right)}\left|u_{\epsilon}\right|^{p_{2}} d x\right)^{1 / p_{2}} \\
= & o_{\epsilon}(1)
\end{aligned}
$$


where $1 / p_{1}+1 / p_{2}=1$. Here we use the facts $u_{\epsilon} \rightarrow 0$ strongly in $L^{q}(\Omega)$ for any $q>1$ and $e^{\left(32 \pi^{2}-\epsilon\right) u_{\epsilon}^{2}}$ is bounded in $L^{s}\left(\Omega \backslash \mathbb{B}_{r}\left(x_{0}\right)\right)$ for some $s>1$. By the definition of $b_{\epsilon}$, we have

$$
\int_{\mathbb{B}_{r}\left(x_{0}\right)} \frac{1}{\lambda_{\epsilon}} b_{\epsilon}\left|u_{\epsilon}\right| e^{\left(32 \pi^{2}-\epsilon\right) u_{\epsilon}^{2}} d x \leq 1 .
$$

Hence

$$
\left|\int_{\mathbb{B}_{r}\left(x_{0}\right)}\left(\phi(x)-\phi\left(x_{0}\right)\right) \frac{b_{\epsilon} u_{\epsilon}}{\lambda_{\epsilon}} e^{\left(32 \pi^{2}-\epsilon\right) u_{\epsilon}^{2}} d x\right| \leq \sup _{x \in \mathbb{B}_{r}\left(x_{0}\right)}\left|\phi(x)-\phi\left(x_{0}\right)\right| .
$$

We immediately derive

$$
\lim _{r \rightarrow 0} \lim _{\epsilon \rightarrow 0} \int_{\Omega}\left(\phi(x)-\phi\left(x_{0}\right)\right) \frac{b_{\epsilon} u_{\epsilon}}{\lambda_{\epsilon}} e^{\left(32 \pi^{2}-\epsilon\right) u_{\epsilon}^{2}} d x=0 .
$$

On the other hand, we can easily get

$$
\begin{gathered}
\lim _{\epsilon \rightarrow 0} \int_{\Omega} \phi\left(x_{0}\right) \frac{b_{\epsilon} u_{\epsilon}}{\lambda_{\epsilon}} e^{\left(32 \pi^{2}-\epsilon\right) u_{\epsilon}^{2}} d x=\phi\left(x_{0}\right) \lim _{\epsilon \rightarrow 0} \frac{\int_{\Omega} u_{\epsilon} e^{\left(32 \pi^{2}-\epsilon\right) u_{\epsilon}^{2}} d x}{\int_{\Omega}\left|u_{\epsilon}\right| e^{\left(32 \pi^{2}-\epsilon\right) u_{\epsilon}^{2}} d x}=\mu \phi\left(x_{0}\right), \\
\lim _{\epsilon \rightarrow 0} \int_{\Omega} b_{\epsilon} u_{\epsilon} \phi d x=\int_{\Omega} G \phi d x \\
\lim _{\epsilon \rightarrow 0}\left(\sum_{i=1}^{\ell} \sum_{j=1}^{n_{i}} \int_{\Omega} \frac{\beta_{i j, \epsilon}}{\lambda_{\epsilon}} b_{\epsilon} e_{i j} \phi d x\right)=\mu \sum_{i=1}^{\ell} \sum_{j=1}^{n_{i}} e_{i j}\left(x_{0}\right) \int_{\Omega} e_{i j} \phi d x .
\end{gathered}
$$

These estimates lead to

$$
\begin{gathered}
\int_{\Omega} \phi\left(\frac{b_{\epsilon} u_{\epsilon}}{\lambda_{\epsilon}} e^{\left(32 \pi^{2}-\epsilon\right) u_{\epsilon}^{2}}+\alpha b_{\epsilon} u_{\epsilon}-\sum_{i=1}^{\ell} \sum_{j=1}^{n_{i}} \frac{\beta_{i j, \epsilon}}{\lambda_{\epsilon}} b_{\epsilon} e_{i j}\right) d x \\
=\mu \phi\left(x_{0}\right)+\alpha \int_{\Omega} G \phi d x-\mu \sum_{i=1}^{\ell} \sum_{j=1}^{n_{i}} e_{i j}\left(x_{0}\right) \int_{\Omega} e_{i j} \phi d x .
\end{gathered}
$$

Therefore, we obtain

$$
\left\{\begin{array}{l}
\Delta^{2} G-\alpha G=\mu\left(\delta_{x_{0}}-\sum_{i=1}^{\ell} \sum_{j=1}^{n_{i}} e_{i j}\left(x_{0}\right) e_{i j}\right) \quad \text { in } \Omega, \\
G=\frac{\partial G}{\partial \nu}=0 \text { on } \partial \Omega .
\end{array}\right.
$$

Take a cut-off function $\eta \in C_{0}^{4}(\Omega)$ such that $\eta \equiv 1$ on $\mathbb{B}_{r}\left(x_{0}\right)$ and $\eta \equiv 0$ on $\Omega \backslash \mathbb{B}_{2 r}\left(x_{0}\right)$, where $\mathbb{B}_{2 r}\left(x_{0}\right) \Subset \Omega$. Let

$$
g(x)=\frac{\mu}{8 \pi^{2}} \eta(x) \log \left|x-x_{0}\right|+G(x) .
$$

Then we have

$$
\left\{\begin{array}{lll}
\Delta^{2} g=f & \text { in } & \Omega, \\
g=\frac{\partial g}{\partial \nu}=0 & \text { on } & \partial \Omega
\end{array}\right.
$$


in a distributional sense, where

$$
\begin{aligned}
f(x)= & -\frac{\mu}{8 \pi^{2}}\left(\Delta^{2} \eta \log \left|x-x_{0}\right|+2 \nabla \Delta \eta \nabla \log \left|x-x_{0}\right|+2 \Delta \eta \Delta \log \left|x-x_{0}\right|\right. \\
& \left.+2 \nabla \eta \nabla \Delta \log \left|x-x_{0}\right|+2 \Delta\left(\nabla \eta \nabla \log \left|x-x_{0}\right|\right)\right)+\alpha G-\mu \sum_{i=1}^{\ell} \sum_{j=1}^{n_{i}} e_{i j}\left(x_{0}\right) e_{i j} .
\end{aligned}
$$

Note that $f$ is bounded in $L^{t}(\Omega)$ for any $t>1$. By the standard regularity theory, we obtain $g \in C^{3}(\bar{\Omega})$. Set $A_{x_{0}}=g\left(x_{0}\right)$ and

$$
v(x)=g(x)-g\left(x_{0}\right)+\frac{\mu}{8 \pi^{2}}(1-\eta) \log \left|x-x_{0}\right| .
$$

Then we get

$$
G(x)=-\frac{\mu}{8 \pi^{2}} \log \left|x-x_{0}\right|+A_{x_{0}}+v(x),
$$

where $A_{x_{0}}$ is a constant depending on $\alpha$ and $x_{0}, v(x) \in C^{3}(\bar{\Omega})$ and $v\left(x_{0}\right)=0$. Since $u_{\epsilon} \in E_{\ell}^{\perp}$, then

$$
\int_{\Omega} G e_{i j} d x=\lim _{\epsilon \rightarrow 0} \int_{\Omega} b_{\epsilon} u_{\epsilon} e_{i j} d x=0, \quad 1 \leq j \leq n_{i}, 1 \leq i \leq \ell .
$$

We have

$$
G \in E_{\ell}^{\perp}
$$

\section{An upper bound}

In this section, we will give an upper bound of the integral $\int_{\Omega} e^{32 \pi^{2} u^{2}} d x$. The proof is based on the Pohozaev type identity and the capacity estimates.

Set $\Omega^{\prime}=\mathbb{B}_{r}\left(x_{\epsilon}\right), y=x_{\epsilon}, u=u_{\epsilon}$ and $f\left(u_{\epsilon}\right)=\frac{1}{\lambda_{\epsilon}} u_{\epsilon} e^{\left(32 \pi^{2}-\epsilon\right) u_{\epsilon}^{2}}+\alpha u_{\epsilon}-\sum_{i=1}^{\ell} \sum_{j=1}^{n_{i}} \frac{\beta_{i j, \epsilon}}{\lambda_{\epsilon}} e_{i j}$. Then we have

$$
F\left(u_{\epsilon}\right)=\frac{e^{\left(32 \pi^{2}-\epsilon\right) u_{\epsilon}^{2}}}{2\left(32 \pi^{2}-\epsilon\right) \lambda_{\epsilon}}+\frac{\alpha}{2} u_{\epsilon}^{2}-\sum_{i=1}^{\ell} \sum_{j=1}^{n_{i}} \frac{\beta_{i j, \epsilon}}{\lambda_{\epsilon}} e_{i j} u_{\epsilon} .
$$

Applying Lemma2, we get for any fixed $r>0$

$$
\begin{aligned}
\int_{\mathbb{B}_{r}\left(x_{\epsilon}\right)} e^{\left(32 \pi^{2}-\epsilon\right) u_{\epsilon}^{2}} d x= & -\frac{\alpha\left(32 \pi^{2}-\epsilon\right) \lambda_{\epsilon}}{4 b_{\epsilon}^{2}} \int_{\mathbb{B}_{r}\left(x_{\epsilon}\right)}\left(b_{\epsilon} u_{\epsilon}\right)^{2} d x+\frac{32 \pi^{2}-\epsilon}{2 b_{\epsilon}} \sum_{i=1}^{\ell} \sum_{j=1}^{n_{i}} \int_{\mathbb{B}_{r}\left(x_{\epsilon}\right)} \beta_{i j, \epsilon} e_{i j} b_{\epsilon} u_{\epsilon} d x \\
& +\frac{r}{4} \int_{\partial \mathbb{B}_{r}\left(x_{\epsilon}\right)} e^{\left(32 \pi^{2}-\epsilon\right) u_{\epsilon}^{2}} d \sigma+\frac{\alpha\left(32 \pi^{2}-\epsilon\right) \lambda_{\epsilon}}{4 b_{\epsilon}^{2}} r \int_{\partial \mathbb{B}_{r}\left(x_{\epsilon}\right)}\left(b_{\epsilon} u_{\epsilon}\right)^{2} d \sigma \\
& -\frac{32 \pi^{2}-\epsilon}{2 b_{\epsilon}} r \sum_{i=1}^{\ell} \sum_{j=1}^{n_{i}} \int_{\partial \mathbb{B}_{r}\left(x_{\epsilon}\right)} \beta_{i j, \epsilon} e_{i j} b_{\epsilon} u_{\epsilon} d \sigma \\
& +\frac{\left(32 \pi^{2}-\epsilon\right) \lambda_{\epsilon}}{4 b_{\epsilon}^{2}}\left(r \int_{\partial \mathbb{B}_{r}\left(x_{\epsilon}\right)}\left|\Delta\left(b_{\epsilon} u_{\epsilon}\right)\right|^{2} d \sigma-4 \int_{\partial \mathbb{B}_{r}\left(x_{\epsilon}\right)} \frac{\partial\left(b_{\epsilon} u_{\epsilon}\right)}{\partial v} \Delta\left(b_{\epsilon} u_{\epsilon}\right) d \sigma\right) \\
& -\frac{\left(32 \pi^{2}-\epsilon\right) \lambda_{\epsilon}}{2 b_{\epsilon}^{2}} r \int_{\partial \mathbb{B}_{r}\left(x_{\epsilon}\right)}\left(2 \frac{\partial\left(b_{\epsilon} u_{\epsilon}\right)}{\partial v} \frac{\partial\left(\Delta\left(b_{\epsilon} u_{\epsilon}\right)\right)}{\partial v}-\nabla \Delta\left(b_{\epsilon} u_{\epsilon}\right) \nabla\left(b_{\epsilon} u_{\epsilon}\right)\right) d \sigma
\end{aligned}
$$


Letting $\epsilon \rightarrow 0$, we have

$$
\begin{aligned}
\lim _{\epsilon \rightarrow 0} \int_{\mathbb{B}_{r}\left(x_{\epsilon}\right)} e^{\left(32 \pi^{2}-\epsilon\right) u_{\epsilon}^{2}} d x= & 16 \pi^{2} \lim _{\epsilon \rightarrow 0} \frac{\lambda_{\epsilon}}{b_{\epsilon}^{2}}\left(\frac{r}{2} \int_{\partial \mathbb{B}_{r}\left(x_{\epsilon}\right)}|\Delta G|^{2} d \sigma-2 \int_{\partial \mathbb{B}_{r}\left(x_{\epsilon}\right)} \frac{\partial G}{\partial v} \Delta G d \sigma\right. \\
& \left.-2 r \int_{\partial \mathbb{B}_{r}\left(x_{\epsilon}\right)} \frac{\partial G}{\partial v} \frac{\partial(\Delta G)}{\partial v} d \sigma+r \int_{\partial \mathbb{B}_{r}\left(x_{\epsilon}\right)} \nabla \Delta G \nabla G d \sigma+o_{r}(1)+o_{\epsilon, r}(1)\right),
\end{aligned}
$$

where $o_{\epsilon, r}(1)$ means $\lim _{\epsilon \rightarrow 0} o_{\epsilon, r}(1)=0$ for any fixed $r>0$ and $o_{r}(1)$ denotes $\lim _{r \rightarrow 0} o_{r}(1)=0$. By straightforward calculation, we obtain

$$
\begin{array}{cc}
\int_{\partial \mathbb{B}_{r}\left(x_{0}\right)}|\Delta G|^{2} d \sigma=\frac{\mu^{2}}{8 \pi^{2} r}+o(1), & \int_{\partial \mathbb{B}_{r}\left(x_{0}\right)} \frac{\partial G}{\partial v} \Delta G d \sigma=\frac{\mu^{2}}{16 \pi^{2}}+o(1), \\
\int_{\partial \mathbb{B}_{r}\left(x_{0}\right)} \frac{\partial G}{\partial \nu} \frac{\Delta G}{\partial \nu} d \sigma=-\frac{\mu^{2}}{8 \pi^{2} r}+o(1), & \int_{\partial \mathbb{B}_{r}\left(x_{0}\right)} \nabla \Delta G \nabla G d \sigma=-\frac{\mu^{2}}{8 \pi^{2} r}+o(1) .
\end{array}
$$

Therefore, we have

$$
\lim _{r \rightarrow 0} \lim _{\epsilon \rightarrow 0} \int_{\mathbb{B}_{r}\left(x_{0}\right)} e^{\left(32 \pi^{2}-\epsilon\right) u_{\epsilon}^{2}} d x=\mu^{2} \lim _{\epsilon \rightarrow 0} \frac{\lambda_{\epsilon}}{b_{\epsilon}^{2}} .
$$

Since $\left|\Delta u_{\epsilon}\right|^{2} \rightarrow \delta_{x_{0}}$ in the sense of measure, we get

$$
\lim _{r \rightarrow 0} \lim _{\epsilon \rightarrow 0} \int_{\Omega \backslash \mathbb{B}_{r}\left(x_{0}\right)} e^{\left(32 \pi^{2}-\epsilon\right) u_{\epsilon}^{2}} d x=|\Omega| .
$$

By these two identities we have

$$
\lim _{\epsilon \rightarrow 0} \int_{\Omega} e^{\left(32 \pi^{2}-\epsilon\right) u_{\epsilon}^{2}} d x=|\Omega|+\mu^{2} \lim _{\epsilon \rightarrow 0} \frac{\lambda_{\epsilon}}{b_{\epsilon}^{2}}
$$

provided that $\mu^{2}>0$. Similar as that in ([25], Lemma 4.6), we have $\mu=1$. The proof is omitted. Hence, (30) can be restated as

$$
\left\{\begin{array}{l}
\Delta^{2} G-\alpha G=\delta_{x_{0}}-\sum_{i=1}^{\ell} \sum_{j=1}^{n_{i}} e_{i j}\left(x_{0}\right) e_{i j} \quad \text { in } \quad \Omega, \\
G=\frac{\partial G}{\partial v}=0 \quad \text { on } \quad \partial \Omega .
\end{array}\right.
$$

Moreover, $G$ can be represented as

$$
G(x)=-\frac{1}{8 \pi^{2}} \log \left|x-x_{0}\right|+A_{x_{0}}+v(x)
$$

where $A_{x_{0}}$ is a constant depending on $x_{0}$ and $\alpha, v(x) \in C^{3}(\bar{\Omega})$ and $v\left(x_{0}\right)=0$.

We now point out the following results:

$$
\lim _{\epsilon \rightarrow 0} \vartheta=\lim _{\epsilon \rightarrow 0} \frac{c_{\epsilon}}{b_{\epsilon}}=1
$$

and $\lambda_{\epsilon} / c_{\epsilon}^{2}$ is bounded. The reader can refer to [25, 30] for details. In particular, $b_{\epsilon}$ can be replaced by $c_{\epsilon}$ in (28). Namely, $c_{\epsilon} u_{\epsilon} \rightarrow G$ in $C_{\text {loc }}^{4}\left(\Omega \backslash\left\{x_{0}\right\}\right)$.

The technique of capacity estimates was first applied to deal with first derivatives of Moser inequality [19]. Slightly modified the proof in ([30], Section 4) which is adapted from the idea of ([25], Section 5), we have

Lemma 4. For any $u \in E_{\ell}^{\perp}$ with $\|u\|_{2, \alpha} \leq 1$, there holds

$$
\sup _{u \in E_{\ell}^{\perp},\|u\|_{2, \alpha} \leq 1} \int_{\Omega} e^{32 \pi^{2} u^{2}} d x \leq|\Omega|+\frac{\pi^{2}}{6} e^{\frac{5}{3}+32 \pi^{2} A_{x_{0}}} .
$$




\section{Test function computation}

In this section, we will construct a sequence of text functions $\phi_{\epsilon}^{*} \in E_{\ell}^{\perp}$ such that $\left\|\phi_{\epsilon}^{*}\right\|_{2, \alpha}=1$ and

$$
\int_{\Omega} e^{32 \pi^{2} \phi_{\epsilon}^{* 2}} d x>|\Omega|+\frac{\pi^{2}}{6} e^{\frac{5}{3}+32 \pi^{2} A_{x_{0}}}
$$

for $\epsilon>0$ sufficiently small. This leads to a contraction with (32). Hence, blow-up can not occur and thus $c_{\epsilon}$ must be bounded. The standard regularity theory leads to the existence of the desired extremal function. The proof of Theorem 1 is completely finished.

To prove (33), we write $r=\left|x-x_{0}\right|$. Recall that $G(x)=-\frac{1}{8 \pi^{2}} \log \left|x-x_{0}\right|+A_{x_{0}}+v(x)$. Set

$$
\phi_{\epsilon}= \begin{cases}c+\frac{a-\frac{1}{16 \pi^{2}} \log \left(1+\frac{\pi r^{2}}{\sqrt{6 c^{2}}}\right)+A_{x_{0}}+v+b r^{2}}{c}, & r \leq R \epsilon, \\ \frac{G}{c}, & r>R \epsilon,\end{cases}
$$

where $a, b$ and $c$ are constants of $\epsilon$ to be determined later, $R=-\log \epsilon$. In order to assure that $\phi_{\epsilon} \in W_{0}^{2,2}(\Omega)$, we require

$$
\lim _{r \rightarrow(R \epsilon)^{-}} \phi_{\epsilon}=\lim _{r \rightarrow(R \epsilon)^{+}} \phi_{\epsilon}
$$

and

$$
\lim _{r \rightarrow(R \epsilon)^{-}} \nabla \phi_{\epsilon}=\lim _{r \rightarrow(R \epsilon)^{+}} \nabla \phi_{\epsilon}
$$

Then we have

$$
\left\{\begin{array}{l}
a=-c^{2}-\frac{\log (R \epsilon)}{8 \pi^{2}}+\frac{\log \left(1+\frac{\pi}{\sqrt{6} R^{2}}\right)}{16 \pi^{2}}-\frac{1}{16 \pi^{2}\left(1+\frac{\pi}{\sqrt{6}} R^{2}\right)}, \\
b=-\frac{1}{16 \pi^{2} R^{2} \epsilon^{2}\left(1+\frac{\pi}{\sqrt{6}} R^{2}\right)} .
\end{array}\right.
$$

A straightforward calculation shows

$$
\int_{\Omega}\left|\Delta \phi_{\epsilon}\right|^{2} d x-\alpha \int_{\Omega} \phi_{\epsilon}^{2} d x=\frac{1}{c^{2}}\left(-\frac{1}{8 \pi^{2}} \log \epsilon+\frac{1}{16 \pi^{2}} \log \frac{\pi}{\sqrt{6}}+A_{x_{0}}-\frac{5}{96 \pi^{2}}+O\left(\frac{1}{\log ^{2} \epsilon}\right)\right) .
$$

Setting $\left\|\phi_{\epsilon}\right\|_{2, \alpha}=1$, we obtain

$$
c^{2}=-\frac{\log \epsilon}{8 \pi^{2}}+\frac{\log \pi}{16 \pi^{2}}-\frac{\log 6}{32 \pi^{2}}-\frac{5}{96 \pi^{2}}+A_{x_{0}}+O\left(\frac{1}{\log ^{2} \epsilon}\right) .
$$

We calculate on $\mathbb{B}_{R \epsilon}\left(x_{0}\right)$

$$
32 \pi^{2} \phi_{\epsilon}^{2} \geq \log \frac{\pi^{2}}{6 \epsilon^{4}}-4 \log \left(1+\frac{\pi r^{2}}{\sqrt{6} \epsilon^{2}}\right)+\frac{5}{3}+32 \pi^{2} A_{x_{0}}+O\left(\frac{1}{\log ^{2} \epsilon}\right) .
$$

Hence

$$
\begin{aligned}
\int_{\mathbb{B}_{R \epsilon}\left(x_{0}\right)} e^{32 \pi^{2} \phi_{\epsilon}^{2}} d x & =\frac{\pi^{2}}{6 \epsilon^{4}} e^{\frac{5}{3}+32 \pi^{2} A_{x_{0}}} \int_{\mathbb{B}_{R \epsilon}\left(x_{0}\right)}\left(1+\frac{\pi}{\sqrt{6}} \frac{r^{2}}{\epsilon^{2}}\right)^{-4} d x \\
& =\frac{\pi^{2}}{6} e^{\frac{5}{3}+32 \pi^{2} A_{x_{0}}}\left(1+O\left(\frac{1}{\log ^{2} \epsilon}\right)\right) .
\end{aligned}
$$


On the other hand, we get on $\Omega \backslash \mathbb{B}_{R \epsilon}\left(x_{0}\right)$

$$
\begin{aligned}
\int_{\Omega \backslash \mathbb{B}_{R \epsilon}\left(x_{0}\right)} e^{32 \pi^{2} \phi_{\epsilon}^{2}} d x & \geq \int_{\Omega \backslash \mathbb{B}_{R \epsilon}\left(x_{0}\right)}\left(1+32 \pi^{2} \phi_{\epsilon}^{2}\right) d x \\
& =|\Omega|+32 \pi^{2} \frac{\|G\|_{2}^{2}}{c^{2}}+O\left(\frac{1}{\log ^{2} \epsilon}\right) .
\end{aligned}
$$

Combining (34) and 35, we conclude

$$
\int_{\Omega} e^{32 \pi^{2} \phi_{\epsilon}^{2}} d x \geq|\Omega|+\frac{\pi^{2}}{6} e^{\frac{5}{3}+32 \pi^{2} A_{x_{0}}}+\frac{32 \pi^{2}}{c^{2}}\|G\|_{2}^{2}+O\left(\frac{1}{\log ^{2} \epsilon}\right) .
$$

Recalling that $\left(e_{i j}\right)\left(1 \leq j \leq n_{i}, 1 \leq i \leq \ell\right)$ is a basis of $E_{\ell}^{\perp}$ verifying (9), we set

$$
\widetilde{\phi}_{\epsilon}=\phi_{\epsilon}-\sum_{i=1}^{\ell} \sum_{j=1}^{n_{i}}\left(\phi_{\epsilon}, e_{i j}\right) e_{i j}
$$

where

$$
\left(\phi_{\epsilon}, e_{i j}\right)=\int_{\Omega} \phi_{\epsilon} e_{i j} d x
$$

Obviously $\widetilde{\phi}_{\epsilon} \in E_{\ell}^{\perp}$. A straightforward calculation gives

$$
\begin{aligned}
\left(\phi_{\epsilon}, e_{i j}\right)= & \int_{\mathbb{B}_{R_{\epsilon}}\left(x_{0}\right)}\left(c+\frac{a-\frac{1}{16 \pi^{2}} \log \left(1+\frac{\pi r^{2}}{\sqrt{6} \epsilon^{2}}\right)+A_{x_{0}}+v+b r^{2}}{c}\right) e_{i j} d x \\
& +\int_{\Omega \backslash \mathbb{B}_{R_{\epsilon}}\left(x_{0}\right)} \frac{G}{c} e_{i j} d x=o\left(\frac{1}{\log ^{2} \epsilon}\right) .
\end{aligned}
$$

Here we have used (31) to obtain

$$
\int_{\Omega \backslash \mathbb{B}_{R_{\epsilon}}\left(x_{0}\right)} \frac{G}{c} e_{i j} d x=-\int_{\mathbb{B}_{R_{\epsilon}}\left(x_{0}\right)} \frac{G}{c} e_{i j} d x=O\left(\epsilon^{4}(-\log \epsilon)^{9 / 2}\right)=o\left(\frac{1}{\log ^{2} \epsilon}\right) .
$$

In view of (37) and the fact $\left\|\phi_{\epsilon}\right\|_{2, \alpha}^{2}=1$, we obtain

$$
\begin{gathered}
\widetilde{\phi}_{\epsilon}=\phi_{\epsilon}+o\left(\frac{1}{\log ^{2} \epsilon}\right), \\
\left\|\widetilde{\phi}_{\epsilon}\right\|_{2, \alpha}^{2}=1+o\left(\frac{1}{\log ^{2} \epsilon}\right) .
\end{gathered}
$$

Combining (36), (38) and (39), we derive

$$
\begin{aligned}
& \int_{\Omega} e^{32 \pi^{2} \frac{\widetilde{\phi}_{\epsilon}^{2}}{\left\|\vec{\phi}_{\epsilon}\right\|_{2, \alpha}^{2}}} d x=\int_{\Omega} e^{32 \pi^{2} \phi_{\epsilon}^{2}+o\left(\frac{1}{\log \epsilon}\right)} d x \\
& \geq\left(1+o\left(\frac{1}{\log \epsilon}\right)\right)\left(|\Omega|+\frac{\pi^{2}}{6} e^{\frac{5}{3}+32 \pi^{2} A_{x_{0}}}+\frac{32 \pi^{2}}{c^{2}}\left(\|G\|_{2}^{2}+o(1)\right)\right) \\
& \geq|\Omega|+\frac{\pi^{2}}{6} e^{\frac{5}{3}+32 \pi^{2} A_{x_{0}}}+32 \pi^{2} \frac{\|G\|_{2}^{2}}{c^{2}}+o\left(\frac{1}{c^{2}}\right) . \\
& 16
\end{aligned}
$$


Set $\phi_{\epsilon}^{*}=\widetilde{\phi}_{\epsilon} /\left\|\widetilde{\phi}_{\epsilon}\right\|_{2, \alpha}$. Noting that $\widetilde{\phi}_{\epsilon} \in E_{\ell}^{\perp}$, we get $\phi_{\epsilon}^{*} \in E_{\ell}^{\perp}$. Moreover $\left\|\phi_{\epsilon}^{*}\right\|_{2, \alpha}=1$ and (33) holds. The contradiction between (32) and (33) shows that $c_{\epsilon}$ must be bounded. This completes the proof of Theorem 1 .

Acknowledgements. This work was partly supported by the Natural Science Foundation of the Education Department of Anhui Province (KJ2016A641) and the Outstanding Young Talents Program of the Education Department of Anhui Province (gxyq2018160).

\section{References}

[1] Adams, A sharp inequality of J. Moser for high order derivatives, Ann. of Math. 128 (1988) 365-398.

[2] Adimurthi, O. Druet, Blow-up analysis in dimension 2 and a sharp form of Moser-Trudinger inequality, Comm. Partial Differential Equations 29 (2004) 295-322.

[3] Adimurthi, K. Sandeep, A singular Moser-Trudinger embedding and its applications, Nonlinear Differ. Equ. Appl. 13 (2007) 585-603.

[4] Adimurthi, Y. Yang, An interpolation of Hardy inequality and Trudinger-Moser inequality in $\mathbb{R}^{N}$ and its applications, Int. Math. Res. Notices 13 (2010) 2394-2426.

[5] T. Aubin, Sru la function exponentielle, C. R. Acad. Sci. Paris, Series A 270 (1970) 1514-1514.

[6] H. Brezis, Functional analysis, Sobolev space and PDEs, Springer, 2011.

[7] P. Cherrier, Une inégalité de Sobolev sur les variétés Riemanniennes, Bull. Sc. Math. 103 (1979) 353-374.

[8] P. Cherrier, Cas d'exception du théorème d'inclusion de Sobolev sur les variétés Riemanniennes et applications, Bull. Sc. Math. 105 (1981) 235-288.

[9] L. Carleson, A. Chang, On the existence of an extremal function for an inequality of J. Moser, Bull. Sci. Math. 110 (1986) 113-127.

[10] G. Csató, P. Roy, Extremal functions for the singular Moser-Trudinger inequality in 2 dimensions, Calc. Var. Partial Differential Equations 54 (2015) 2341-2366.

[11] A. Dall' Acqua, G. Sweeis, Estimates for Greem function and Possion kernels of higher order Dirichlet boundary value problems, J. Differential Equations 205 (2004) 466-487.

[12] M. de Souza, J. M. do Ó, A sharp Turdinger-Moser type inequality in $\mathbb{R}^{2}$, Trans. Amer. Math. Soc. 366 (2014) 4513-4549.

[13] W. Ding, J. Jost, J. Li, G. Wang, The differential equation $-\Delta u=8 \pi-8 \pi h e^{u}$ on a compact Riemann surface, Asian J. Math. 1 (1997) 230-248.

[14] J. M. do Ó, M. de Souza, A sharp inequality of Truding-Moser type and extremal funcions in $H^{1, n}\left(\mathbb{R}^{n}\right)$, J. Differential Equations 258 (2015) 4062-4101.

[15] M. Flucher, Extremal functions for Trudinger-Moser inequality in 2 dimensions, Comment. Math. Helv. 67 (1992) 471-497.

[16] L. Fontana, Sharp borderline Sobolev inequalities on compact Riemannian manifolds, Comment. Math. Helv. 68 (1993) 415-454

[17] X. Li, An improved singular Trudinger-Moser inequality in $\mathbb{R}^{N}$ and its entremal functions, J. Math. Anal. Appl. 462 (2018), 1109-1129.

[18] X. Li, Y. Yang, Extremal functions for singular Trudinger-Moser inequalities in the entire Euclidean space, J. Differential Equations 264 (2018) 4901-4943.

[19] Y. Li, Moser-Trudinger inequality on compact Riemannian manifolds of dimension two, J. Partial Differential Equations 14 (2001) 163-192.

[20] Y. Li, The extremal functions for Moser-Trudinger inequality on compact Riemannian manifolds, Sci. China Series A. Math. 48 (2005) 618-648.

[21] Y. Li, C. Ndiaye, Extremal functions for Moser-Trudinger type inequality on compact closed 4-manifolds, J. Geom. Anal. 17 (2001) 669-699.

[22] Y. Li, B. Ruf, A sharp Trudinger-Moser type inequality for unbounded domains in $\mathbb{R}^{N}$, Indiana Univ. Math. J. 57 (2008) 451-480.

[23] C. Lin, A classification of solutions of conformally invariant fourth order equation in $R^{n}$, Comment. Math. Helv. 73 (1998) 206-231.

[24] K. Lin, Extremal functions for Moser's inequality, Trans. Amer. Math. Soc. 348 (1996) 2663-2671

[25] G. Lu, Y. Yang, Adams' inequalities for bi-Laplacian and extremal functions in dimension four, Adv. Maths. 220 (2009) 1135-1170. 
[26] G. Lu, Y. Yang, The sharp constant and extremal functions for Moser-Trudinger inequalities involving $L^{p}$ norms, Discrete and Continuous Dynamical Systems 25 (2009) 963-979.

[27] G. Mancini, K. Sandeep, Moser-Trudinger inequality on conformal discs, Commun. Contemp. Math. 12 (2010) 1055-10687.

[28] E. Mitidieri, A Rellich type identity and applications, Comm. Partial Differential Equations 18 (1993) 125-151.

[29] J. Moser, A sharp form of an inequality by N.Trudinger, Ind. Univ. Math. J. 20 (1971) 1077-1091.

[30] H. Nguyen, A sharp Adams inequality in dimension four and its extremal functions, arXiv: 1701.08249, 2017.

[31] S. Pohozaev, The Sobolev embedding in the special case $p l=n$, Proceedings of the technical scientific conference on advances of scientific reseach 1964-1965, Mathematics sections, 158-170, Moscov. Energet. Inst., Moscow, 1965.

[32] B. Ruf, A sharp Trudinger-Moser type inequality for unbounded domains in $\mathbb{R}^{2}$, J. Funct. Anal. 219 (2005) 340 367.

[33] C. Tintarev, Trudinger-Moser inequality with remainder terms, J. Funct. Anal. 266 (2014) 55-66.

[34] N. Trudinger, On embeddings into Orlicz spaces and some applications, J. Math. Mech. 17 (1967) 473-484.

[35] J. Wei, X. Xu, Classification of solutions of higher order conformally invariant equations, Math. Ann. 313 (1999) 207-228.

[36] Y. Yang, A sharp form of Moser-Trudinger inequality in high dimension, J. Funct. Anal. 239 (2006) 100-126.

[37] Y. Yang, A sharp form of the Moser-Trudinger inequality on a compact Riemannian surface, Trans. Amer. Math. Soc. 359 (2007) 5761-5776.

[38] Y. Yang, Trudinger-Moser inequalities on complete noncompact Riemannian manifolds, J. Funct. Anal. 263 (2012) 1894-1938

[39] Y. Yang, Extremal functions for Trudinger-Moser inequalities of Adimurthi-Druet type in dimension two, J. Differential Equations 258 (2015) 3161-3193.

[40] Y. Yang, A Trudinger-Moser inequality on a compact Riemannian surface involving Gaussian curvature, J. Geom. Anal. 26 (2016) 2893-2913

[41] Y. Yang, X. Zhu, Blow-up analysis concerning singular Trudinger-Moser inequalities in dimension two, J. Funct. Anal. 272 (2017) 3347-3374

[42] V. I. Yudovich, Some estimates connected with integral operators and with solutions of elliptic equations, Sov. Math. Docl. 2 (1961) 746-749.

[43] J. Zhu, Improved Moser-Trudinger inequality involving $L^{p}$ norm in $n$ dimensions, Adv. Nonlinear Study 14 (2014) 273-293. 